Maria Silvia Petty Moutinho

SIMONE ELIAS ${ }^{2}$

CLÁUDIO KEMP ${ }^{3}$

Afonso Celso Pinto NAZÁrio ${ }^{4}$

EDMUND CHADA BaRACAT ${ }^{5}$

Artigos originais

Palavras-chave

Mamografia

Mama/lesões

Mama/cirurgia

Neoplasias mamárias/cirurgia Biopsia por agulha

Técnicas estereotáxicas

Keywords

Mammography/methods

Breast/lesions

Breast neoplasms/surgery

Breast/surgery

Biopsy needle

Stereotaxic techniques

Correspondêncic

Simone Elios

Rua Botucatu, 527 - Vila Clementino CEP 04023-061 - São Paulo/SP Fone: 5575-3451/Fax: 3083-6755 E-mail: simoneelias3@yahoo.com.br

Recebido

14/08/2007

Aceito com modificacõos $12 / 12 / 2007$

\section{Acurácia diagnóstica da biópsia percutânea com agulha grossa orientada por estereotaxia nas lesões mamárias categoria BI-RADS ${ }^{\circledR} 4$}

\author{
Diagnostic accuracy of stereotactic core-needle biopsy of non-palpable \\ breast lesions categorized as $B I-R A D S^{\circledR} 4$
}

\section{Resumo}

OBJETIVO: avaliar a acurácia da biópsia com agulha grossa (BAG) orientada por estereotaxia nas lesões mamárias categoria BI-RADS ${ }^{\circledR}$ 4. MÉTODOS: realizou-se uma análise retrospectiva das lesões categoria BI-RADS ${ }^{\circledR} 4$ que foram submetidas à BAG orientada por estereotaxia no período de junho de 1998 a junho de 2003. As pacientes com resultados benignos, mas com baixa suspeição à imagem radiográfica, foram orientadas a acompanhamento mamográfico. As pacientes com resultados malignos ou com lesões especiais (atipias e lesões papilíferas) foram submetidas ao tratamento padronizado. Aquelas com resultado benigno, mas que apresentavam imagem mamográfica de maior risco, foram encaminhadas para biópsia cirúrgica (BC). Foram considerados padrão-ouro (1) o acompanhamento radiográfico inalterado por, no mínimo, três anos daquelas lesões de baixa suspeição que resultaram benigno na BAG e (2) os resultados das biópsias cirúrgicas, quando essas foram realizadas (nas BAG cujos resultados foram malignos ou benignos com imagem de maior risco). Foram realizados os cálculos estatísticos de sensibilidade, especificidade e valor preditivo positivo e negativo do método. RESULTADOS: das 118 lesões não palpáveis categoria BI-RADS ${ }^{\circledast} 4$ submetidas à BAG orientada por estereotaxia, 27 casos correspondiam à doença maligna, 81 eram benignas e 10 eram lesões com atipia ou papilíferas. Para a análise estatística, foram selecionados 108 pacientes (excluídas as lesões com atipia e papilíferas). A sensibilidade da BAG foi de $87,1 \%$ e a especificidade foi de $100 \%$. $O$ valor preditivo positivo foi de $100 \%$ e o negativo, de 95, 1\%. A acurácia da BAG nessa casuística foi de 96,3\%. A taxa de falso-negativo foi de 3,7\% (4/108). A prevalência de diagnóstico de malignidade nas lesões BI-RADS ${ }^{\circledR} 4$ nessa amostra foi de 29,7\% (31 / 1 18). CONCLUSÕES: a biópsia estereotáxica com agulha grossa mostrou-se uma alternativa segura à BC. Quando seu resultado é positivo, orienta o planejamento terapêutico, e, quando negativo (em imagens radiográficas de baixa suspeição), permite o acompanhamento.

Abstract

PURPOSE: to assess the accuracy (rate of correct predictions) of stereotactic core needle biopsy (CNB) of risk category BI-RADS ${ }^{\circledR} 4$ breast lesions. METHODS: a retrospective analysis of category BI-RADS ${ }^{\circledR} 4$ breast lesions that had been submitted to a stereotactic core-needle biopsy from June 1998 to June 2003. Patients with histological benign results consistent with the radiographic image were referred to mammographic follow-up. Patients with malign diagnosis and papillary lesions were submitted to standard specific treatment. Excisional biopsies were performed when results were benign, but in disagreement with the mammographic image. It was considered as a gold-standard attendance: (1) the mammographic follow-up of low suspicion lesions with benign results at CNB, which stayed unchanged for, at least, three years, and (2) surgical resection when specimen results were malign or benign, but with a high suspicion on mammography. Sensitivity (S) specificity (E) and overall accuracy of stereotactic CNB were statistically analyzed. RESULTS: among the 118 non-palpable lesions of category BI-RADS ${ }^{\circledR} 4$ submitted to CNB, the results obtained were: 27 malign cases, 81 benign, and ten lesions with atypical or papillary lesions. The statistical analysis comprised 108
Trabalho realizado na disciplina de Mastologia do Departamento de Ginecologia da Escola Paulista de Medicina da Universidade Federal de São Paulo - UNIFESP - São Paulo (SP), Brasil.

Pós-graduanda da Disciplina de Mastologia do Departamento de Ginecologia da Escola Paulista de Medicina da Universidade Federal de São Paulo - UNIFESP - São Paulo (SP), Brasil.

2 Colaboradora da Disciplina de Mastologia do Departamento de Ginecologia da Escola Paulista de Medicina da Universidade Federal de São Paulo - UNIFESP - São Paulo (SP), Brasil.

${ }^{3}$ Doutor, Professor Adjunto do Departamento de Ginecologia da Escola Paulista de Medicina da Universidade Federal de São Paulo - UNIFESP - São Paulo (SP), Brasil.

4 Professor Associado do Departamento de Ginecologia da Escola Paulista de Medicina da Universidade Federal de São Paulo - Unifesp/ EPM - São Paulo (SP), Brasil.

${ }_{5}$ Professor Titular do Departamento de Ginecologia da Universidade de São Paulo - USP - São Paulo (SP), Brasil; Professor Titular do Departamento de Ginecologia da Escola Paulista de Medicina da Universidade Federal de São Paulo - UNIFESP - São Paulo (SP), Brasil. Os autores declaram que não há conflitos de interesse. 
patients (atypical and papillary lesions were excluded). CNB sensitivity was $87.1 \%$ and specificity $100 \%$. The positive predictive value was $100 \%$ and the negative, $95.1 \%$. False negatives occurred in 3.7\% (4/108) of cases. The prevalence of malign diagnostics in the BI-RADS ${ }^{\circledR} 4$ lesions of this sample was 29.7 (31/1 18). The accuracy of this method in this casuistic was 96.3\%. CONCLUSIONS: these results support stereotactic CNB as an extremely reliable alternative to open biopsy, in the diagnosis and definition of breast lesions. In positive results, it is possible to indicate the appropriate therapy, and, in negative (when mammography shows low suspicion), it allows a follow up.

\section{Introdução}

Não é mais discutido o valor do rastreamento mamográfico para detecção precoce do câncer de mama. Porém, apesar de todo incremento tecnológico, a mamografia continua sendo um exame de alta sensibilidade e moderada especificidade, pois o valor preditivo positivo (VPP) varia de 15 a $38 \%{ }^{1}$. Assim, para cada 1.000 mamografias de rastreamento realizadas, 20 a 40 biópsias são indicadas ${ }^{2,3}$ e, destas, apenas 20 a $35 \%$ são carcinomas ${ }^{4}$. Cerca de 120.000 biópsias são realizadas anualmente nos Estados Unidos em lesões não palpáveis detectadas pela mamografia ${ }^{4,5}$. Esse fato provocou um aumento de $20 \%$ nos custos dos programas de rastreamento ${ }^{5}$.

Por muitos anos, a biópsia cirúrgica (BC) foi o único método para remoção de áreas suspeitas à mamografia para análise anatomopatológica. Na década de 1970, a biópsia com agulha fina de lesões não palpáveis orientada pela imagem foi introduzida por Nördenstrom, do Instituto Karolinska (Estocolmo, Suécia) e tornouse rapidamente um padrão no manejo das lesões não palpáveis da mama; porém, seus excelentes resultados não conseguiram ser reproduzidos em outros centros. Ainda na Suécia, Lindgrën desenvolveu uma pistola automática, que, acoplada a um trocater, possibilitava a obtenção de fragmentos de tecidos de lesões de forma rápida e eficiente ${ }^{6}$.

Nos Estados Unidos, Parker et al. ${ }^{6}$ adaptaram o dispositivo de Lindgrën para um sistema de biópsia estereotáxica com a paciente sentada. Paralelamente, a mesa de estereotaxia que permitia realizar esse procedimento com a paciente deitada, desenvolvido na Suécia, foi adaptada para acomodar o propulsor modificado por Parker ${ }^{7}$.

A biópsia com agulha grossa (BAG) tornou-se, assim, um método alternativo à $\mathrm{BC}$, por ser minimamente invasiva, não requerer internação hospitalar, ser realizado com anestesia local, deixar cicatrizes discretas e ter poucas complicações ou intercorrências ${ }^{6}$.

Numerosos estudos, com maior ou menor número de casos, têm mostrado que a BAG é método seguro com custo inferior à BC, sendo uma opção confiável na prática clínica. Quando seu diagnóstico é positivo, esse procedimento apresenta alta especificidade, porém, quando resultados negativos são obtidos, esses devem ser interpretados com cuidado. As características da lesão a ser investigada, o calibre da agulha, a quantidade de fragmentos e a localização da lesão na mama estão entre os principais fatores que devem ser considerados para a escolha adequada do método, com o objetivo de diminuir o risco de resultados falsos-negativos ou subestimados. Deve-se considerar, ainda, a prevalência do câncer de mama na população estudada ${ }^{8-10}$.

O objetivo deste trabalho foi avaliar a acurácia da BAG orientada por estereotaxia nas lesões não palpáveis da mama, classificadas na categoria Breast Imaging Reporting and Data System (BI-RADS $\left.{ }^{\boxplus}\right)^{4}$.

\section{Métodos}

No ambulatório da disciplina de Mastologia do Departamento de Ginecologia da Universidade Federal de São Paulo (Unifesp), o sistema BI-RADS ${ }^{\otimes}$ foi adotado desde 1998 para a classificação das lesões mamográficas não palpáveis. Assim, os exames mamográficos que apresentaram conclusão diagnóstica nas categorias 4 (achados mamográficos suspeitos) e 5 (achados mamográficos altamente suspeitos) vêm sendo habitualmente submetidos a investigação anatomopatológica, que se tornou conduta habitual nessas investigações.

Para esse estudo, realizou-se, então, uma análise retrospectiva das lesões mamográficas categoria BI-RADS ${ }^{\circledR} 4$, que foram submetidas à $\mathrm{BAG}$ orientadas por estereotaxia no período de junho de 1998 a junho de 2003.

Esse estudo foi previamente aprovado pelo Comitê de Ética em Pesquisa da instituição. Todas as pacientes que foram submetidas aos procedimentos recebiam previamente orientação e assinavam termo de consentimento pós-informado sobre o mesmo.

Foram selecionadas 118 pacientes, com idade variando entre 40 a 75 anos (média de 56 anos), cujos exames foram realizados com a técnica adequada: a lesão foi alcançada pela agulha; a quantidade de fragmentos obtida foi suficiente (mínimo de cinco para nódulos ou assimetrias, e de sete para microcalcificações) ${ }^{7,11}$; e, quando a lesão era constituída de calcificações, sua presença foi confirmada na radiografia dos fragmentos ${ }^{12}$.

A maioria das lesões foi representada por microcalcificações (104), seguidas de nódulos (12) e assimetria focal (2). 
As causas de exclusão foram as limitações inerentes à dificuldade técnica do procedimento (radiografia do fragmento sem partículas, lesões profundas, próximas à parede torácica ou mamas volumosas), relacionadas ao tipo da lesão (microcalcificações muito tênues de difícil visualização) ou relacionadas às pacientes (lipotímia, sangramento excessivo) ${ }^{7}$.

As BAG dirigidas por estereotaxia foram realizadas em um mamógrafo MD 4000 Philips (Mammoguide: Mamo Diagnost 4000, Philips Medical Systems, The Netherlands) associado à unidade de estereotaxia. Para a realização das biópsias desse estudo, foram utilizadas agulhas de calibre 12, adaptadas a um propulsor automático com cursor de 2,2 cm (Biopty, Bard Urological, Covington, GA). Esse propulsor consiste basicamente em uma caixa metálica que comporta um sistema de molas seqüenciais, no qual se introduz um trocater. Com o disparo, uma agulha biselada cortante avança através do tecido e, em seguida, um dispositivo cilíndrico externo efetua o corte, apreendendo o tecido na chanfradura da primeira agulha ${ }^{7}$.

O procedimento iniciou-se pela escolha da melhor incidência mamográfica para atingir a lesão e, posteriormente, eram obtidas incidências anguladas (angulando-se o tubo de raios $\mathrm{X} \mathrm{a}+15^{\circ}$ e $-15^{\circ}$. O equipamento realizava um cálculo trigonométrico para a obtenção das coordenadas $\mathrm{X}, \mathrm{Y}$ e $\mathrm{Z}$ da lesão. Após anti-sepsia e uma pequena incisão na pele, introduzia-se o trocater. Após o primeiro disparo, uma incidência radiográfica confirmava a posição adequada da agulha em relação à lesão. A próxima etapa era, então, a obtenção dos fragmentos ${ }^{7,8}$.

Após a realização das biópsias, sistematicamente realizou-se a correlação entre a classificação da imagem das lesões e o diagnóstico histológico obtido.

As pacientes com resultados benignos concordantes com a imagem radiográfica (lesões que, apesar de classificadas na categoria BI-RADS 4, eram da baixa suspeição) foram orientadas a acompanhamento mamográfico unilateral em seis meses, bilateral em 12 meses, seguidos de controle anual por, no mínimo, 36 meses. Quando a lesão permaneceu inalterada por esse período, foi considerada como sendo resultado verdadeiro-negativo do procedimento. As pacientes com resultados malignos ou com lesões especiais (atipias e lesões papilíferas) foram encaminhadas para tratamento de acordo com a conduta padronizada na disciplina.

Os diagnósticos anatomopatológicos foram divididos em três categorias: maligno: carcinoma ductal in situ, carcinoma ductal invasivo, carcinoma lobular in situ e carcinoma lobular invasivo; benigno: alterações fibrocísticas, fibrose, fibroadenoma e hiperplasia sem atipia; e lesões especiais: hiperplasia ductal com atipia e lesões papilíferas.

Os cálculos estatísticos de sensibilidade, especificidade e valor preditivo positivo e negativo (VPN) foram realizados excluindo-se as lesões com atipias e papilíferas. Essas lesões apresentam características próprias e são consideradas como especiais e, até o momento, constituem-se em limitações das biópsias percutâneas. Esses casos totalizaram 10 pacientes nessa casuística (sete casos de atipia e três de lesões papilíferas).

\section{Resultados}

Das 118 lesões não palpáveis, categoria BI-RADS ${ }^{\circledR} 4$, submetidas à BAG orientada por estereotaxia, obtiveram-se 27 casos malignos, 81 benignos e dez lesões especiais (atipia ou papilíferas). Para cálculo dos resultados estatísticos, foram utilizadas 108 pacientes (excluídas as lesões especiais), distribuídas conforme o resultado da BAG como benigno ou maligno e seus respectivos resultados definitivos (BC ou seguimento clínico estável por período mínimo de 36 meses), de acordo com a Tabela 1.

Os 27 casos com resultados anatomopatológicos malignos foram encaminhados para tratamento de acordo com o protocolo da disciplina. Todos os resultados malignos obtidos com a BAG foram confirmados na BC $(\mathrm{VPP}=100 \%)$. Não houve casos falsos-positivos, conforme se observa na Tabela 2, que relaciona os casos malignos obtidos na BAG com a BC.

Tabela 1 - Avaliação dos resultados anatomopatológicos das biópsias com agulha grossa e resultados definitivos*.

\begin{tabular}{lrccc|}
\hline & \multicolumn{4}{c}{ Resultado definitivo } \\
\cline { 2 - 5 } Biópsia com agulla grossa & \multicolumn{3}{c}{ Maligno } & \multicolumn{2}{c}{ Benigno } \\
\cline { 2 - 5 } & $\mathbf{n}$ & $\%$ & $\mathrm{n}$ & $\%$ \\
\hline Maligno & 27 & 25 & & 0 \\
Benigno & 4 & 3,7 & 77 & 71,3 \\
Total (n=108) & 31 & 28,7 & 77 & 71,3 \\
\hline
\end{tabular}

*Biópsias cirúrgicas ou seguimento clínico inalterado (mínimo de 36 meses).

Tabela 2 - Correlação entre os resultados positivos (malignos) da biópsia com agulha grossa e seus respectivos anatomopatológicos na biópsia cirúrgica ( $n=27)$.

\begin{tabular}{lccc}
\hline \multirow{2}{*}{ Biópsia com agulha grossa } & \multicolumn{3}{c}{ Biópsia cirúrgica } \\
\cline { 2 - 4 } & CDI & CDIS & CLI \\
\hline Carcinoma ductal invasivo (CDI) & 13 & & \\
Carcinoma ductal in situ (CDI) & 7 & 6 & \\
Carcinoma lobular invasivo (CDI) & & & 1 \\
\hline
\end{tabular}


Observa-se que todos os casos de lesões invasivas $(\mathrm{n}=14)$ foram confirmados pela BC (13 carcinomas ductais invasivos e um caso de carcinoma lobular invasivo). No caso de lesões in situ ( $\mathrm{n}=13)$, em seis foram confirmadas lesões in situ e em sete o resultado definitivo da cirurgia foi carcinoma ductal invasivo.

Nas lesões em que a BAG resultou em lesão benigna, todas as imagens radiológicas foram novamente avaliadas e revisadas para definir a conduta final. Quando as imagens radiográficas eram de menor suspeição, optouse por seguimento clínico-radiológico, o que ocorreu em $77,7 \%$ (63/81). Esse seguimento variou entre $36 \mathrm{e}$ 60 meses (com média de 48 meses).

Durante esse período, quatro pacientes apresentaram alteração da lesão em seu controle mamográfico, tendo sido então submetidas à BC. Em todos os casos, o resultado benigno da BAG foi confirmado. As alterações na morfologia da lesão inicial foram observadas no segundo ano de acompanhamento em dois casos e, após 24 meses, nos outros dois. Nenhum caso sofreu alteração no primeiro ano de seguimento.

As pacientes cujo resultado da BAG foi lesão benigna, mas a imagem mamográfica radiológica era de maior suspeição, foram encaminhadas à BC. Os resultados anatomopatológicos destas foram negativos (benignos) em 14 casos e quatro apresentaram resultados malignos (Tabela 3). Esses quatro casos constituíram-se nos resultados falsos-negativos da BAG.

A sensibilidade foi de $87,1 \%$; a especificidade, $100 \%$; o VPP, 100\%; o VPN, 95,1\%. A taxa de falso-negativo foi de $3,7 \%$ (4/108). A acurácia do método (proporções de predições corretas) nessa casuística foi de 96,3\%.

Foram excluídos da análise estatística dez casos de lesões especiais: sete eram lesões com atipia (os resultados na BC foram: três casos de alterações fibrocísticas, um caso de hiperplasia com atipia, dois casos de carcinoma invasivo e uma paciente em seguimento por falta de condições cirúrgicas) e três eram lesões papilíferas (cuja

Tabela 3 - Correlação dos resultados anatomopatológicos das biópsias com agulha grossa versus biópsia cirúrgica os casos discordantes $(\mathrm{n}=18)$.

\begin{tabular}{lcccccc}
\hline & \multicolumn{5}{c}{ Resultados anatomopatológicos } \\
\cline { 2 - 6 } & \multicolumn{5}{c}{ BAG } & \multicolumn{3}{c}{ Biópsia cirúrgica } \\
\cline { 2 - 6 } & Adenose & FA & Benignos & CLI & CDIS & CDI \\
\hline Adenose & 2 & & & 1 & 1 \\
AFC & & & 2 & & & \\
FA & & 3 & & & 1 & \\
HT & & & & & & \\
Fibrose & & & & 1 & & \\
Benignos & & & 7 & & \\
\hline
\end{tabular}

Obs.: os casos falsos-negativos estão grifados e em negrito.

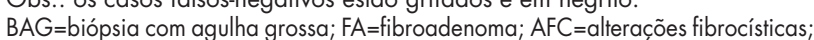
$\mathrm{HT}=$ hiperplasia sem atipia; $\mathrm{CLI}=$ carcinoma lobular invasivo; $C D I S=$ carcinoma ductal in situ; $\mathrm{CDI}=$ carcinoma ductal invasivo.
$\mathrm{BC}$ revelou um papiloma, um carcinoma papilífero in situ e o outro caso também não tinha condições cirúrgicas).

Assim, a prevalência de câncer de mama na população total com lesões mamográficas categoria BI-RADS ${ }^{\boxplus}$ foi de 29,7\% (31/118).

Nenhuma paciente apresentou complicações imediatas ou graves decorrentes do procedimento. Ocorreram dois casos de hematoma (sem necessidade de drenagem) e não houve casos de infecção. A queixa principal foi o desconforto do exame.

\section{Discussão}

O padrão-ouro na avaliação das anormalidades mamográficas é a BC precedida da localização por agulhamento. Embora esse procedimento seja, de fato, efetivo, a porcentagem de casos nos quais esse método falha em remover a lesão pode variar entre 2 e $20 \%{ }^{13,14}$.

Em revisão de 17 trabalhos sobre o tema, envolvendo 3.418 pacientes, mostrou-se um índice de falha de cerca de $3 \%$. Diferentes motivos podem ser apontados: reparo inadequado da agulha, deslocamento da mesma antes ou durante a cirurgia, falta de radiografia do espécime para confirmar a retirada da lesão e microcalcificações localizadas na pele ${ }^{13}$.

A BAG foi, então, introduzida como sendo um método menos invasivo, de menor custo e de boa acurácia para avaliar lesões detectadas na mamografia. Em um estudo que incluiu mais de 6.000 biópsias por agulha demonstrou-se que esse procedimento era reprodutível e confiável, constituindo-se em alternativa à biópsia tradicional ${ }^{15}$.

Sugere-se que a melhor indicação para BAG seria obter diagnóstico anatomopatológico em mulheres que mais provavelmente não têm câncer, mas para as quais os achados da imagem colocamnas como necessitando de biópsia ${ }^{16}$. De fato, as pacientes portadoras de lesões BI-RADS ${ }^{\circledR} 4$ são as que mais se beneficiam deste método, pois se pode evitar a BC em lesões benignas e aumentar o número de diagnósticos de câncer em lesões que deverão ser submetidas à cirurgia. No entanto, essas pacientes devem ser adequadamente acompanhadas quando o resultado de suas biópsias por agulha for benigno ${ }^{17}$.

O tempo de seguimento sugerido para confirmar a benignidade do achado é de no mínimo dois anos e, preferencialmente, de três anos ${ }^{17}$. Em estudo que inclui 379 pacientes com diagnóstico benigno à $\mathrm{BAG}$ em lesões mamárias não palpáveis, todas foram acompanhadas por até seis anos. Concluiu-se que pacientes nessa condição devem ser acompanhadas após a BAG 
Quadro 1 - Taxas de resultados falsos-negativos das biópsias com agulha grossa encontradas na literatura.

\begin{tabular}{|c|c|c|}
\hline Autor & Ano & Taxa de falsos-negativos (\%) \\
\hline Parker ef al. ${ }^{15}$ & 1994 & 1,1 \\
\hline Jackman et al..$^{20}$ & 1999 & 1,9 \\
\hline Lee et al..21 & 1997 & 0,9 \\
\hline Adler et al. ${ }^{22}$ & 2000 & 5 \\
\hline Burns ef al. ${ }^{14}$ & 2000 & 4,7 \\
\hline White ef al. ${ }^{23}$ & 2001 & 3,2 \\
\hline Acheson ef al. ${ }^{18}$ & 2002 & 0,3 \\
\hline Ciatto ef alo. ${ }^{12}$ & 2007 & 4,4 \\
\hline
\end{tabular}

em seis e 12 meses, retornando após esse período para controle anual. A taxa de falso-negativo desse estudo foi de $0,3 \%{ }^{18}$. Em nossa amostra, o menor tempo de acompanhamento foi de 36 meses, com média de 48 meses, intervalo que confirma a benignidade das lesões biopsiadas pela BAG.

Outro estudo mostrou que lesões benignas diagnosticadas por meio da BAG podiam ser acompanhadas de modo seguro, pois se encontrara sensibilidade de $100 \%$ e especificidade de 98,9\%, além de VPP de 99,7\% e VPN de $100 \%^{19}$. Na literatura, encontramos taxas de resultados falsos-negativos que variaram de 0,3 a $4,7 \%{ }^{12,14,15,18,20-23}$ (Quadro 1).

Nossa taxa de falso-negativo foi $3,7 \%$. Dentre os quatro casos de falsos-negativos dessa série, três apresentavam lesões extensas na mamografia (microcalcificacões ocupando mais de $2,5 \mathrm{~cm}$ de extensão), o que aumenta a chance de falso-negativo na BAG.

As BAG têm limitações relacionadas ao volume de material coletado. Essa limitação é importante, principalmente nas lesões papilíferas e outras lesões especiais (como a cicatriz radial e o carcinoma lobular), que dependem da retirada quase completa da lesão para sua análise anatomopatológica adequada ${ }^{24,25}$. Resultados como hiperplasia ductal com atipia e carcinoma in situ devem ser considerados como subdiagnóstico, pois são lesões que podem estar associadas a outra de maior gravidade. No caso da BAG, isso ocorre em cerca de 56 e $20 \%$, respectivamente ${ }^{20,25}$. Assim, o achado dessas lesões implica retirada completa por meio de BC.

Um estudo com grande casuística, que incluiu 1.236 lesões submetidas à BAG, avaliou a importância clínica de lesões de alto risco incomuns detectadas por este método. $\mathrm{Na} \mathrm{BC}$, três de 22 lesões papilíferas revelaram-se carcinomas, oito de nove cicatrizes radiais apresentaram atipia e um de cinco carcinomas lobulares in situ apresentava também carcinoma ductal in situ. Concluiu ser prudente a remoção de toda a lesão nos casos de alterações com atipias, lesões papilíferas ou achados não compatíveis ${ }^{26}$.

Quando os resultados de BAG não são definitivamente negativos ou positivos com invasão podem estar associados a resultados subestimados. Identificar os fatores que contribuem para o subdiagnóstico na imagem mamográfica ou nos fragmentos pode reduzir esse índice ${ }^{27}$. Entre esses fatores, encontram-se as lesões especiais; assim, optou-se por excluir essas lesões para a adequada interpretação de nossos resultados.

Outro fator muito importante a ser ressaltado no momento da escolha do método para investigação de uma alteração mamográfica é o custo do procedimento. O impacto do método de biópsia nos custos da investigação diagnóstica mamária é considerável. A $\mathrm{BAG}$, quando comparada à $\mathrm{BC}$, reduz o custo médio do procedimento pela metade ${ }^{6}$.

Atualmente, dispõe-se ainda da mamotomia, método que tem se revelado superior à BAG em muitos aspectos. No entanto, seu custo elevado mostra uma redução em relação à BC de apenas $20 \%{ }^{28}$.

Concluindo, nossos resultados demonstram que a biópsia estereotáxica com agulha grossa é uma alternativa segura à $\mathrm{BC}$ e demonstra a confiabilidade do método, desde que conhecidas suas indicações e limitações, além de respeitadas todas as etapas de sua realização.

\section{Referências}

1. Heywang-Kobrunner SH. Mammography. In: HeywangKobrunner SH, Dershaw DD, Schreer I, editors. Diagnostic breast imaging. Mammography, sonography, magnetic resonance imaging, and interventional procedures. Stuttgart: Thieme; 2001. p. 68-70.

2. King ES, Rimer BK, Seay J, Balshem A, Engstrom PF. Promoting mammography use through progressive interventions: is it effective? Am J Public Health. 1994;84(1):104-6.

3. Kopans DB. The positive predictive value of mammography. AJR Am J Roentgenol. 1992;158(3):521-6.
4. Liberman L, Abramson AF, Squires FB, Glassman JR, Morris EA, Dershaw DD. The breast imaging reporting and data system: positive predictive value of mammographic features and final assessment categories. AJR Am J Roentgenol. 1998;171(1):35-40.

5. Liberman L, Fahs MC, Dershaw DD, Bonaccio E, Abramson AF, Cohen MA, et al. Impact of stereotaxic core breast biopsy on cost of dignosis. Radiology. 1995; 195(3):633-7.

6. Parker SH, Lovin JD, Jobe WE, Luethke JM, Hopper KD, Yakes WF, et al. Stereotactic breast biopsy with a biopsy gun. Radiology. 1990;176(3):741-7. 
7. Kemp C, Baracat FF, Rostagno R. Lesões não palpáveis da mama: diagnóstico e tratamento. Rio de Janeiro: Revinter; 2003.

8. Burbank F, Parker SH. Methods for evaluating the quality of an imageguided breast biopsy program. Semin Breast Dis. 1998;1 (2):71-83.

9. Cipolla C, Fricano S, Vieni S, Amato C, Napoli L, Graceffa G, et al. Validity of needle core in the histological characterisation of mammary lesions. Breast. 2006;15(1):76-80.

10. Houssami N, Ciatto S, Ellis I, Ambrogetti D. Underestimation of malignancy of breast core-needle biopsy: concepts and precise overall and category-specific estimates. Cancer. 2007;109(3):487-95.

11. Koskela AK, Sudah M, Berg MH, Kärjä VJ, Mustonen PK, Kataja $\mathrm{V}$, et al. Add-on device for stereotactic core-needle breast biopsy: how many biopsy specimens are needle for a reliable diagnosis? Radiology. 2005;236(3):801-9.

12. Ciatto $S$, Houssami $N$, Ambrogetti $D$, Bianchi $S$, Bonardi R, Brancato $B$, et al. Accuracy and underestimation of malignancy of breast core needle biopsy: the Florence experience of over 4000 consecutive biopsies. Breast Cancer Res Treat. 2007;101(3):291-7.

13. Kaelin CM, Smith TJ, Homer M, Taback B, Azurin D, Schmid CH, et al. Safety, accuracy, and diagnostic yield of needle localization biopsy of the breast performed using local anesthesia. J Am Coll Surg. 1994;179(3):267-72.

14. Burns PR, Brown JP, Roe SM, Sprouse LR II, Yancey AE, Witherspoon LE. Stereotactic core-needle breast biopsy by surgeons: minimum 2-year follow-up of benign lesions. Ann Surg. 2000;232(4):542-8.

15. Parker SH, Burbank F, Jackman RJ, Aucreman CJ, Cardenosa G, Cink TM, et al. Percutaneous large-core breast biopsy: a multiinstitucional study. Radiology. 1994;193(2):359-64.

16. Logan-Young WW, Janus JA, Destounis SV, Hoffman NY. Appropriate role of core breast biopsy in the management of probably benign lesions. Radiology. 1994;190(2):313.

17. Kopans DB. Caution on core. Radiology. 1994;193(2):325-6.

18. Acheson MB, Patton RG, Howisey RL, Lane RF, Morgan A, Rowbotham RK. Three- to six-year follow-up for 379 benign image-guided large-core needle biopsies of nonpalpable breast abnormalities. J Am Coll Surg. 2002;195(4):462-6.
19. Duncan JL 3rd, Cederbom GJ, Champaign JL, Smetherman DH King TA, Farr $\mathrm{GH}$, et al. Benign diagnosis by image-guided coreneedle breast biopsy. Am Surg. 2000;66(1):5-9.

20. Jackman RJ, Nowels KW, Rodriguez-Soto J, Marzoni FA Jr, Finkelstein SI, Shepard M. Stereotactic, automated, large-core needle biopsy of nonpalpable breast lesions: false negative and histologic underestimate rates after long-term follow-up. Radiology. 1999;210(3):799-805

21. Lee $\mathrm{CH}$, Egglin TK, Philpotts L, Maineiro MB, Tocino I. Costeffectiveness of stereotactic core needle biopsy: analysis by means of mammographic findings. Radiology. 1997;202(3):849-54.

22. Adler DD, Light RJ, Granstrom P, Hunter TB, Hunt KR. Follow-up of benign results of stereotactic core breast biopsy. Acad Radiol. 2000;7(4):248-53.

23. White RR, Halperin TJ, Olson JA Jr, Soo MS, Bentley RC, Seigler HF. Impact of core-needle breast biopsy on the surgical management of mammographic abnormalities. Ann Surg. 2001;233(6):769-77.

24. Liberman L, Dershaw DD, Glassman JR, Abramson AF, Morris EA, LaTrenta LR, et al. Analysis of cancers not diagnosed at stereotactic core breast biopsy. Radiology. 1997;203(1):151-7.

25. Liberman L, Dershaw DD, Rosen PP, Giess CS, Cohen MA, Abramson AF, et al. Stereotaxic core biopsy of breast carcinoma: accuracy at predicting invasion. Radiology. $1995 ; 194(2): 379-81$.

26. Philpotts LE, Shaheen NA, Jain KS, Carter D, Lee CH. Uncommon high-risk lesions of breast diagnosed at stereotactic core-needle biopsy: clinical importance. Radiology. 2000;216(3):831-7.

27. Liberman L. Clinical management issues in percutaneous core breast biopsy. Radiol Clin North Am. 2000;38(4):791-807.

28. Soo MS, Kliewer MA, Ghate S, Helsper RS, Rosen EL. Stereotactic breast biopsy of noncalcified lesions: a cost-minimization analysis comparing 14-gauge multipass automated core biopsy to 14- and 11-gauge vacuum-assisted biopsy. Clin Imaging. $2005 ; 29(1): 26-33$. 